\title{
Note on a Theorem of Hilbert.
}

\author{
By \\ G. H. Hardy.
}

1. It was proved by Hilbert, in the course of his investigations in the theory of integral equations, that the series

$$
\sum_{m, n=1}^{\infty} \frac{a_{m} a_{n}}{m+n} \quad\left(a_{m} \geqq 0\right)
$$

is convergent whenever $\sum a_{m}^{2}$ is convergent. Of this theorem, which is one of the simplest and most beautiful in the theory of double series of positive terms, at least five essentially different proofs have been published. Hilbert's own proof, which depends upon the theory of Fourier's series, is outlined by Weyl in his Inaugural-Dissertation ${ }^{1}$ ). Another proof was given by Wiener"), and two more by $S \operatorname{chur}^{3}$ ); but none of these proofs is as simple and elementary as might be desired. Thus Schur's first proof depends upon the theory of quadratic and bilinear forms in an infinity of variables; and his second (which is unquestionably the most elegant of all) on a change of variables in a double integral. And Wiener's proof, while genuinely elementary, is distinctly artificial.

To these four proofs I added recently') a fifth which seemed to me to lack nothing in simplicity. I observed first that Hilbert's theorem is an immediate corollary of another theorem which seems of some interest in itself. This theorem is as follows:

1) H. Wey1, „Singuläre Integralgleichungen", Göttingen 1908, p. 83.

2) F. W. Wiener, „Elementarer Beweis eines Reihensatzes von Herrn Hilbert", Math. Annalen, 68 (1910), pp. 361-366.

s) I. Schur, „Bemerkungen zur Theorie der beschränkten Bilinearformen mit unendlich vielen Veränderlichen ", Journal für Math., 140 (1912), pp. 1-28.

4) G. H. Hardy, "Notes on some points in the integral caloulus (51)", Messenger of Mathematies, 48 (1918), pp. 107-112. 
Theorem A. If $\sum a_{n}^{2}$ is convergent then

$$
\sum\left(\frac{A_{n}}{n}\right)^{2}
$$

where $A_{n}=a_{1}+a_{2}+\ldots+a_{n}$, is convergent.

In order'to deduce Hilbert's theorem from Theorem $A$, we have only to observe that the convergence of the two series in question involves that of

$$
\sum \underset{n}{a_{n} A_{n}}
$$

and $a$ fortiori of

or of

$$
\sum_{n} a_{n} \sum_{m=1}^{n} \frac{a_{m}}{m+n}
$$

$$
\sum_{m<n} \frac{a_{m} a_{n}}{m+n}
$$

2. Dr. Marcel Ries $z$, to whom recently $I$ communicated Theorem $A$, at once found another proof which is equal to my own in simplicity, and which seems to both of us more natural and therefore preferable. His proof naturally suggests an interesting generalisation, viz:

Theorem B. If $x>1$ and $\sum a_{n}^{*}$ is convergent, then

$$
\sum\left(\frac{A_{n}}{n}\right)^{n}
$$

is convergent.

I give the proof of this theorem here: Riesz's proof of Theorem A is obtained by writing $x=2$ throughout the argument.

Let

$$
\Phi_{n}=n^{-n}+(n+1)^{-x}+(n+2)^{-x}+\ldots
$$

Then

$$
\begin{gathered}
\left.\sum_{1}^{N}\left(\frac{A_{n}}{n}\right)^{*}=\sum_{1}^{N} A_{n}^{*}\left(\Phi_{n}-\Phi_{n+1}\right)=\sum_{1}^{N}\left(A_{n}^{*}-A_{n-1}^{*}\right) \Phi_{n}-A_{N}^{*} \Phi_{N+1}{ }^{*}\right) \\
\leqq \\
\sum_{1}^{N}\left(A_{n}^{*}-A_{n-1}^{*}\right) \Phi_{n} \leqq \varkappa \sum_{1}^{N} a_{n} A_{n}^{x-1} \Phi_{n} .
\end{gathered}
$$

But

$$
\Phi_{n}<n^{-x}+\int_{n}^{\infty} x^{-x} d x=n^{-x}+\frac{n^{-(x-1)}}{x-1} \leqq x \stackrel{x}{-1} n^{-(x-1)}
$$

B) $A_{0}=0$. 
and therefore

$$
\left.\sum_{1}^{N}\left(\frac{A_{n}}{n}\right)^{*} \leqq \frac{x^{*}}{x-1} \sum_{1}^{N} a_{n}\left(\frac{A_{n}}{n}\right)^{x-1} \leqq \frac{x^{21}}{x-1}\left(\sum_{1}^{N} a_{n}^{*}\right)^{\frac{1}{x}}\left\{\sum_{1}^{N}\left(\frac{A_{n}}{n}\right)^{*}\right\}^{\frac{x-1}{x}}\right) .
$$

Hence

$$
\sum_{1}^{N}\left(\frac{A_{n}}{n}\right)^{x} \leqq\left(\frac{x^{2}}{x-1}\right)^{x} \sum_{1}^{N} a_{n}^{x}
$$

which proves the theorem.

3. It would be interesting to know what is the best constant which. can appear on the right hand side of (2). It is certain that the actual constant is unnecessarily large. For

$$
\begin{aligned}
\Phi_{n} & n^{-x}+(n+1)^{-x}+\ldots+(2 n)^{-x}+\ldots+(3 n)^{-x}+\ldots \\
& \left.<n\left\{n^{-x}+(2 n)^{-x}+(3 n)^{-x}+\ldots\right\}=n^{-(x-1)} \zeta(x)^{3}\right) .
\end{aligned}
$$

If we use (3) instead of (1), we are led to an inequality in which the constant $\left(\frac{x^{2}}{x-1}\right)^{*}$ is replaced by the smaller constant

$$
\{x \zeta(x)\}^{*}
$$

The corresponding inequality for integrals is

$$
\int_{a}^{\infty}\left(\frac{F}{x}\right)^{x} d x \leqq\left(\frac{x}{x-1}\right)^{x} \int_{a}^{\infty} f^{x} d x
$$

o) By the well known inequality

$$
\sum a b \leq\left(\sum a^{x}\right)^{\frac{x}{x}}\left(\sum b^{\frac{x}{x-1}}\right)^{\frac{x-1}{x}}
$$

which seems to be due to Hölder: see E. Landau, "Cher einen Konvergenzsatz", Göttinger Nachrichten, 1907, pp. 25-27.

7) I have taken this simple argument from a letter of Prof. Sehur, my own proot of the inequality having been unnecessarily complioated. Prof. Sohur also points out to me that, when $x=2$, the constant $\left(\frac{x^{2}}{x-1}\right)^{x}=16$ in (2) oun certainly be replaced by $\left(\begin{array}{c}x \\ x-1\end{array}\right)^{x}=4$; and that this is an almost immediate deduction from the inequality

$$
\sum_{1}^{N}\left(1-\begin{array}{c}
2 \\
n+1
\end{array}\right)\left(\frac{A_{n}}{n}\right)^{2} \leqq 4 \sum_{1}^{N} a_{n ?}^{2}
$$

proved in my note in the Messenger of Mathematics.

Prof. Schurs letter contains a number of other interesting developwents, which I hope may be made public before long. 
Here

$$
F=\int_{a}^{x} f d t
$$

and $a$ and $f$ are positive. It will be observed that in this inequality we have a smaller constant than in (1), and it is easy to show, by taking $f=x^{-\frac{1}{x}-\varepsilon}$, where $\varepsilon$ is small, that the constant is in fact the correct one. I can show that, in (2), the constant cannot be replaced by any smaller constant than that which occurs in (4); but whether this constant will suffice $I$ cannot say.

4. Theorems of this character naturally suggest numerous generalisations. I content myself with mentioning the following theorem, which may be proved by a slight modification of the proof of Theorem $B$.

Theorem C. If $x>1, \lambda_{n}$ and $a_{n}$ are positive, and $\sum \lambda_{n} a_{n}^{*}$ is convergent, then

$$
\dot{\sum} \lambda_{n}\left(\frac{\lambda_{1} a_{1}+\lambda_{2} a_{2}+\ldots+\lambda_{n} a_{n}}{\lambda_{1}+\lambda_{2}+\ldots+\lambda_{n}}\right)^{n}
$$

is convergent.

Trinity College, Cambridge, 25 October 1919.

(Eingegangen am 30. Oktober 1919.) 